\title{
Waveguiding in surface plasmon polariton band gap structures
}

Bozhevolnyi, S.I.; Østergaard, John Erland; Leosson, Kristjan; Skovgaard, Peter M. W.; Hvam, Jørn Märcher

Published in:

Physical Review Letters

Link to article, DOI:

10.1103/PhysRevLett.86.3008

Publication date:

2001

Document Version

Publisher's PDF, also known as Version of record

Link back to DTU Orbit

Citation $(A P A)$ :

Bozhevolnyi, S. I., Østergaard, J. E., Leosson, K., Skovgaard, P. M. W., \& Hvam, J. M. (2001). Waveguiding in surface plasmon polariton band gap structures. Physical Review Letters, 86(14), 3008-3011.

https://doi.org/10.1103/PhysRevLett.86.3008

\section{General rights}

Copyright and moral rights for the publications made accessible in the public portal are retained by the authors and/or other copyright owners and it is a condition of accessing publications that users recognise and abide by the legal requirements associated with these rights.

- Users may download and print one copy of any publication from the public portal for the purpose of private study or research.

- You may not further distribute the material or use it for any profit-making activity or commercial gain

- You may freely distribute the URL identifying the publication in the public portal

If you believe that this document breaches copyright please contact us providing details, and we will remove access to the work immediately and investigate your claim 


\title{
Waveguiding in Surface Plasmon Polariton Band Gap Structures
}

\author{
Sergey I. Bozhevolnyi* \\ Institute of Physics, Aalborg University, Pontoppidanstraede 103, DK-9220 Aalborg Øst, Denmark \\ John Erland, Kristjan Leosson, Peter M. W. Skovgaard, and Jørn M. Hvam \\ Research Center COM, Technical University of Denmark, Building 345v, DK-2800 Kongens Lyngby, Denmark
}

(Received 26 October 2000)

\begin{abstract}
Using near-field optical microscopy, we investigate propagation and scattering of surface plasmon polaritons (SPP's) excited in the wavelength range of 780-820 $\mathrm{nm}$ at nanostructured gold-film surfaces with areas of 200-nm-wide scatterers arranged in a 400-nm-period triangular lattice containing line defects. We observe the SPP reflection by such an area and SPP guiding along line defects at $782 \mathrm{~nm}$, as well as significant deterioration of these effects is $815 \mathrm{~nm}$, thereby directly demonstrating the SPP band gap effect and showing first examples of SPP channel waveguides in surface band gap structures.
\end{abstract}

DOI: $10.1103 /$ PhysRevLett.86.3008

Conventional photonic band gap (PBG) structures are composed of regions with a periodic modulation of the refractive index that do not allow the propagation of electromagnetic waves in a certain interval of wavelengths, i.e., that exhibit the PBG effect [1]. From the point of view of solid state physics, the periodicity in a dielectric constant results in the photonic stop band just like the periodicity in an electrostatic potential gives rise to the forbidden electronic energy band [2]. Using the language of physical optics, the PBG effect can be viewed as an extension of Bragg reflection in one direction (e.g., occurring in lasers with distributed feedback) to Bragg reflection of waves propagating in any direction. The PBG effect (in periodic media) can also be regarded as a counterpart of strong (Anderson) localization of light in random media [3] both being interference phenomena related to strong multiple scattering of light. Recently, the interest in the PBG structures (photonic crystals) has been further fueled by the theoretical predictions [4] and experimental demonstration (albeit in the millimeter range of wavelengths) [5] of efficient waveguiding around a sharp corner of a line defect in the PBG structure. It became clear that the PBG structures, when properly designed and realized, might be advantageously used for photonic circuits allowing for an unprecedented level of integration [6]. First examples of successfully realized two-dimensional (2D), PBG-based, optical components include a defect mode laser [7], microcavities [8], highly dispersive elements ("superprisms") [9], and a bent laser cavity [10].

Given the perspective of integrating various PBG-based components within a few hundred micrometers, we realized that one may employ for the same purpose even relatively lossy 2D waves, e.g., surface plasmon polaritons (SPP's). SPP's are surface electromagnetic waves propagating along a metal-dielectric interface and having the amplitudes exponentially decaying in the neighbor media [11]. The SPP propagation constant $\beta$ is determined by the corresponding dielectric constants $\varepsilon_{1}$ and $\varepsilon_{2}$ of the two media: $\beta=(2 \pi / \lambda)\left[\varepsilon_{1} \varepsilon_{2} /\left(\varepsilon_{1}+\varepsilon_{2}\right)\right]^{0.5}$, where $\lambda$
PACS numbers: 42.70.Qs, 07.79.Fc, 42.25.-p, 73.20.Mf

is the wavelength of light in free space. The propagation length of SPP's is thereby limited due to the internal damping (Ohmic losses): $L_{\mathrm{SPP}}=(2 \operatorname{Im} \beta)^{-1}$, where $L_{\mathrm{SPP}}$ is the distance after which the SPP intensity decreases to $1 / e$ of its starting value. For example, the SPP propagation length along a silver-air interface exceeds $100 \mu \mathrm{m}$ for light wavelengths already in the near infrared range [11], implying that the realization of integrated optical circuits based on the SPP band gap (SPPBG) effect is feasible at the telecommunication wavelengths. It should also be mentioned that the SPPBG effect has been observed (though indirectly) by performing angular and wavelength resolved measurements of the reflectivity of a periodically corrugated silver film [12].

In this Letter, we report what we believe to be the first direct observations (with a near-field optical microscope) of the SPPBG effect and SPP guiding along line defects in SPPBG structures. This work represents a further development of our activity within micro-optics of SPP's [13]. However, instead of controlling elastic (in the surface plane) SPP scattering by sets of a few microscatterers [13], we employ elastic SPP scattering within areas of periodically arranged surface scatterers to inhibit the SPP propagation inside these areas for a certain range of wavelengths.

The experimental setup consists of a stand-alone scanning near-field optical microscope (SNOM) [14] and an arrangement for SPP excitation in the usual Kretschmann configuration [11]. SPP's are excited along a 45-nm-thick gold film that has been thermally evaporated on a glass substrate and whose surface has been covered with areas of a 400-nm-period triangular lattice of $\sim 200$-nm-wide and $\sim 45$-nm-high gold scatterers (Fig. 1). This nanopatterning has been produced by using electron beam lithography on a resist layer on the gold film, evaporation of a second gold layer, and liftoff. The fabricated surface structure contained line defects of different widths and two main orientations, viz. $\Gamma K$ [Figs. 1(a) and 1(d)] and $\Gamma M$ [Figs. 1(b) and 1(c)], of the irreducible Brillouin zone of the lattice 


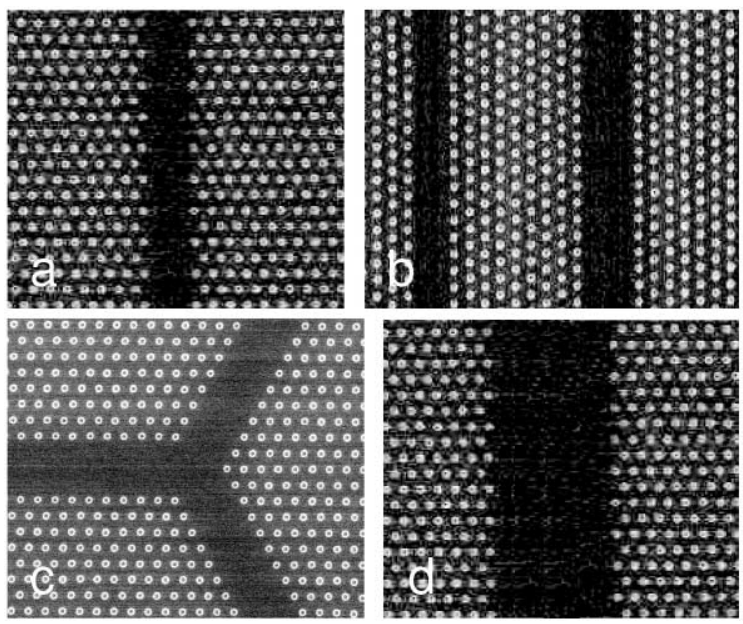

FIG. 1. Scanning electron microscope pictures of different regions of a 400-nm-period triangular lattice with line defects formed in a resist mask layer on a 45-nm-thick gold film. This mask was used to fabricate the actual surface structure and the regions shown (a) $-(d)$ correspond to the surface areas investigated in this work (Figs. 2-5).

[1]. The $p$-polarized light beam from a Ti:sapphire laser ( $\lambda=780-820 \mathrm{~nm}, \quad P \sim 50 \mathrm{~mW}$ ) is weakly focused (focal length $\approx 500 \mathrm{~mm}$, spot size $\sim 300 \mu \mathrm{m}$ ) onto the sample attached with immersion oil to the base of a glass prism. The SPP excitation is recognized as a minimum in the angular dependence of the reflected light power [11,13]. The arrangement allows for an efficient SPP excitation in the range of $780-820 \mathrm{~nm}$, when the angle of incidence is adjusted for the optimum SPP excitation at $800 \mathrm{~nm}$. The SPP propagation length $L_{\mathrm{SPP}}$ evaluated in this range for an air-gold interface $\left(\varepsilon_{1}=\right.$ $\left.1, \varepsilon_{2} \approx-23+1.8 i\right)$ is $\approx 35 \mu \mathrm{m}$ [11]. In the configuration used, it gets further reduced due to the radiative damping (resonant reradiation in the substrate) and inelastic SPP scattering out of the surface plane.

The SPP local field is probed with an uncoated sharp fiber tip that is scanned along the sample surface at a constant distance of a few nanometers maintained by shear force feedback. The radiation scattered by the fiber tip is partially collected by the fiber itself and propagated in the form of fiber modes towards another end of the fiber, whereupon it is detected by a photomultiplier [13]. The SPP excitation exhibited a well-pronounced resonance behavior, and the average optical signal from the fiber was more than 10 times smaller if the incident angle was out of resonance or if the fiber tip was moved $\sim 1 \mu \mathrm{m}$ away from the sample surface. This means that an optical image obtained during the scanning procedure represents mainly the total SPP field intensity distribution along the film surface (and not that of field components scattered out of the surface plane) [13]. Finally, it should be noted that all images (Figs. 1-5) presented here are oriented in the way that the excited SPP propagates upwards in the vertical direction.
Reflection by the periodic surface structure of the resonantly excited SPP propagating approximately along the $\Gamma K$ direction was investigated for different light wavelengths (Fig. 2). The optical images represent the intensity interference patterns formed by the excited SPP, the SPP reflected by the periodic structure, and the SPP's scattered by other surface structures (e.g., markers) located outside of the imaged area. At $\lambda \cong 782 \mathrm{~nm}$, the reflected SPP is close in strength to the excited SPP and much stronger than the scattered SPP's resulting in very bright interference fringes seen in front of the structure [Fig. 2(b)]. This feature along with a virtually zero signal just $\sim 2 \mu \mathrm{m}$ inside the structure indicate its high reflectivity and strong damping of the incident SPP. The reflectivity and damping rapidly decrease with the increase of the light wavelength and the reflected SPP becomes weak, comparable to the scattered SPP's and spread inside the structure. As a result, the difference in the SPP intensity distributions inside and outside of the structure diminishes at $\lambda \cong 792 \mathrm{~nm}$ [Fig. 2(c)] and practically vanishes at $\lambda \cong 815 \mathrm{~nm}$ [Fig. 2(d)]. Such a drastic wavelength dependence of the SPP intensity inside the structure and its reflectivity represents, in our opinion, an unambiguous evidence of the SPPBG effect. Finally, note that the optical images are not visibly affected by the presence of the scatterer in front of the structure, a feature that also indicates that the influence of topographical artifacts is rather small in this configuration [15].
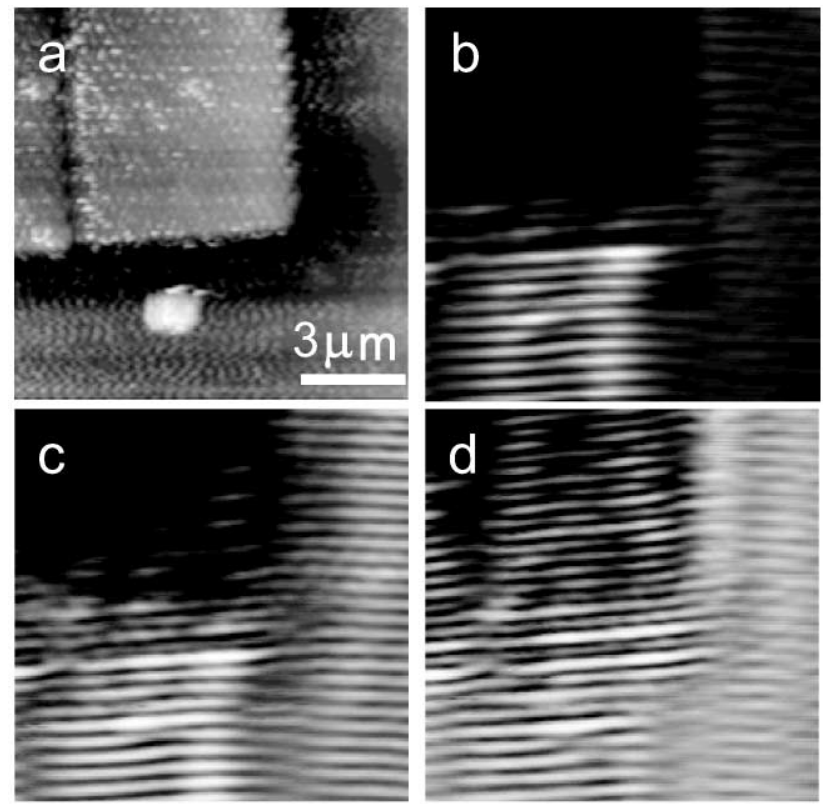

FIG. 2. Gray-scale (a) topographical and near-field optical images $\left(10.5 \times 10.5 \mu \mathrm{m}^{2}\right)$ taken at $\lambda \cong($ b) 782 , (c) 792 , and (d) $815 \mathrm{~nm}$. Periodic surface structure with a line defect having $\Gamma K$ orientation corresponds to the resist structure shown in Fig. 1(a). Depth of the topographical image is $90 \mathrm{~nm}$. Contrast, i.e., the relative difference between maximum and minimum detected optical signal, of the optical images is $\sim 98 \%$. 
Given the occurrence of the SPPBG effect, one may expect to observe, at the corresponding wavelength, the SPP guiding along a line defect in the SPPBG structure. As it turned out, we found no evidence of SPP guiding along the line defect of $\Gamma K$ orientation [Fig. 2(b)] obtained by removing a chain of hexagonal clusters [Fig. 1(a)]. This may seem surprising, but one should bear in mind that very little is known about 2D SPPBG structures on the theoretical side, probably because even the case of SPP scattering by an individual surface defect requires elaborate simulations [16]. We found that the line defect of $\Gamma M$ orientation with nearly the same width (having three rows of scatterers removed) does support the SPP guiding at $782 \mathrm{~nm}$, albeit over only a short distance of $\sim 5 \mu \mathrm{m}$ (Fig. 3). Note the SPPBG structure reflects efficiently the incident SPP at this wavelength but poorly at $792 \mathrm{~nm}$. One can therefore conclude that the SPPBG effect is also present for $Г M$ orientation with the gap being centered close to $782 \mathrm{~nm}$. Topographical and optical cross sections [Fig. 3(d)] taken $\sim 4 \mu \mathrm{m}$ inside the structure indicate that the SPPBG waveguide mode is well confined inside the waveguide, whose width is only $\approx 1.39 \mu \mathrm{m}$ [Fig. 1(b)]. At the same time, the neighboring waveguide (having two rows of scatterers removed and the width of $\approx 1.04 \mu \mathrm{m}$ ) does not support the guided mode even over a short distance. It seems that the width of the latter waveguide is below the cutoff value for the fundamental mode, whereas the width of the for-
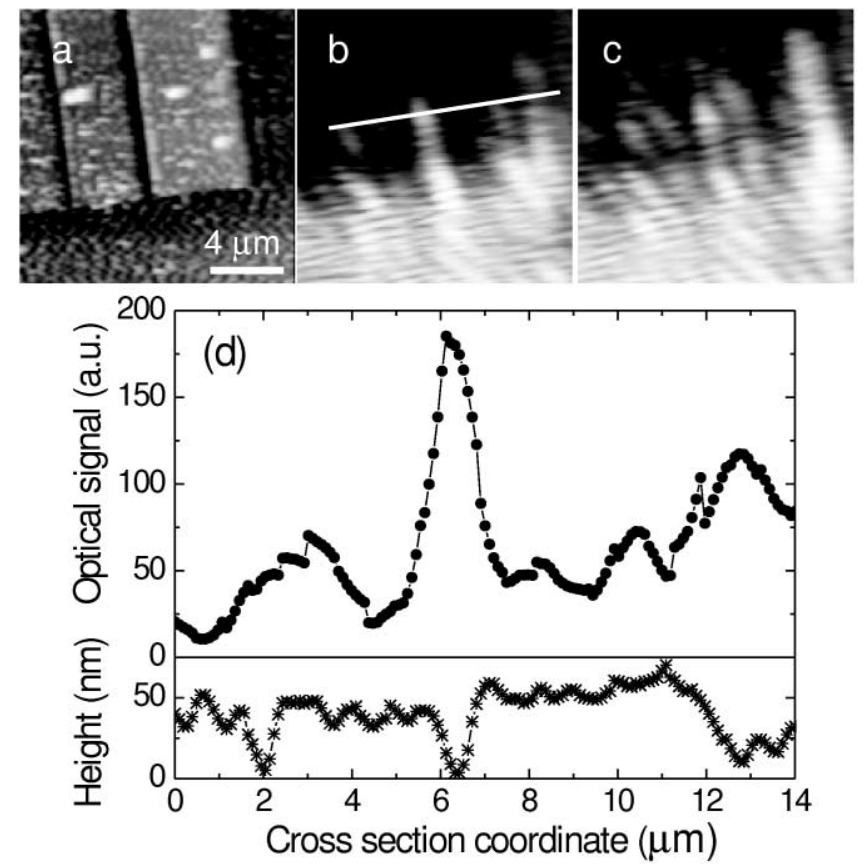

FIG. 3. Gray-scale (a) topographical and near-field optical images $\left(15 \times 15 \mu \mathrm{m}^{2}\right)$ taken at $\lambda \cong$ (b) 782 , (c) $792 \mathrm{~nm}$ together with (d) cross sections along the line marked on the corresponding optical image. Periodic surface structure with two line defects having $\Gamma M$ orientation corresponds to the resist structure shown in Fig. 1(b). Depth of the topographical image is $150 \mathrm{~nm}$. Contrast of the optical images is $\sim 97 \%$. mer is just above it. This can make the mode propagation very sensitive to the waveguide irregularities and account for the short propagation distance observed for this mode [Fig. 3(b)].

The above conjecture is supported by the images obtained with the SPPBG structure of $\Gamma K$ orientation containing the line defect along $\Gamma M$ direction (Fig. 4). One can notice a weak mode (at $782 \mathrm{~nm}$ ) propagating in this defect that also has three rows of scatterers removed [Fig. 1(c)]. In addition, it is seen that a pronounced SPP beam propagates in a wide $(\sim 3.2 \mu \mathrm{m})$ channel (of $\Gamma K$ orientation) between two SPPBG structures [Fig. 1(d)]. Topographical and optical cross sections [Fig. 4(d)] taken $\sim 4 \mu \mathrm{m}$ inside the structure display the intensity profiles of both the (fundamental) mode of the narrow SPPBG waveguide and a combination of (probably the first two) modes of the wide channel. Again, the SPP guiding in the line defects and the SPP reflection of the SPPBG structure disappear when the wavelength increases to $815 \mathrm{~nm}$ [Fig. 4(c)]. The propagation of the combination of modes in the wide SPPBG channel over $\sim 18 \mu \mathrm{m}$ without noticeable loss (Fig. 5) demonstrates the ability of line defects in the SPPBG structures to efficiently guide SPP fields. Note that, contrary to what might have been expected [4,5], the SPP guiding observed thus far (Figs. 4 and 5) does not show the effect of turning around sharp
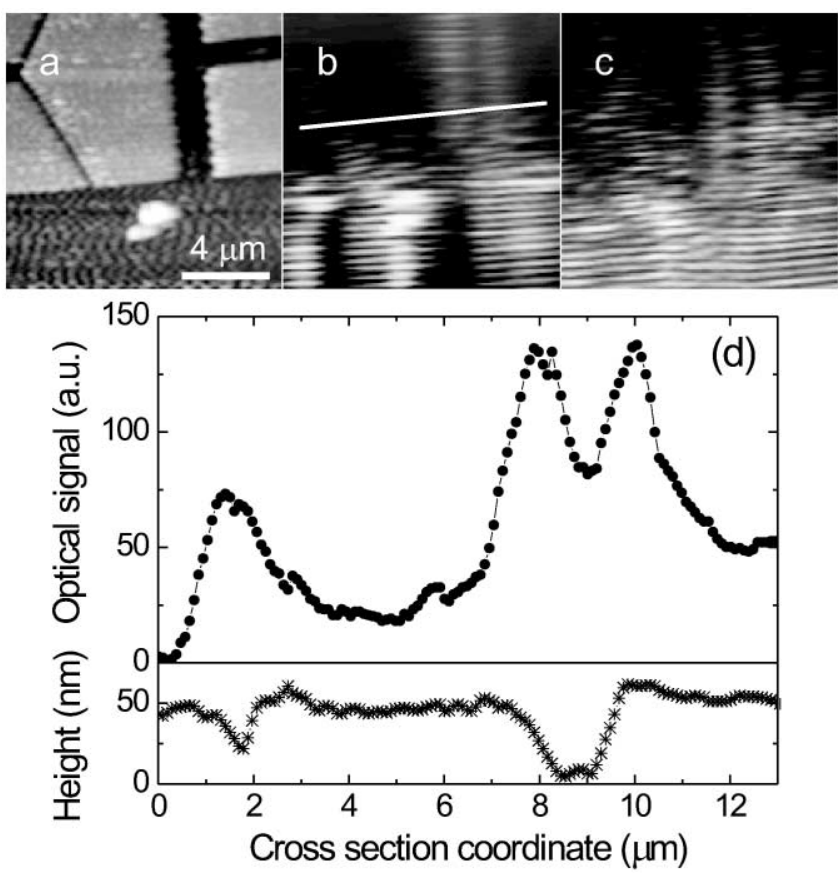

FIG. 4. Gray-scale (a) topographical and near-field optical images $\left(13 \times 13 \mu \mathrm{m}^{2}\right)$ taken at $\lambda \cong$ (b) 782 , and (c) $815 \mathrm{~nm}$ together with (d) cross sections along the line marked on the corresponding optical image. Periodic surface structure with the line defect of $\Gamma M$ orientation corresponds to the resist structure shown in Fig. 1(c), whereas the wide defect of $\Gamma K$ orientation is shown in Fig. 1(d). Depth of the topographical image is $170 \mathrm{~nm}$. Contrast of the optical images is $\sim 98 \%$. 


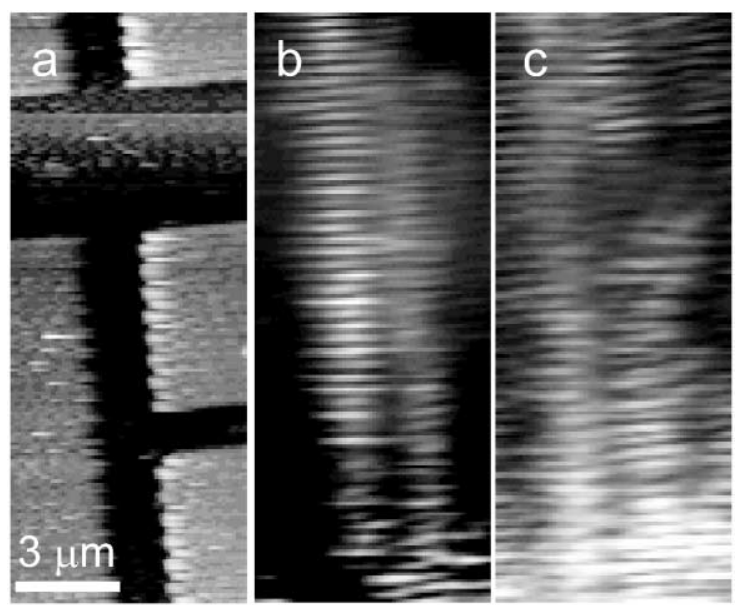

FIG. 5. Gray-scale (a) topographical and near-field optical images $\left(7 \times 17.5 \mu \mathrm{m}^{2}\right)$ taken at $\lambda \cong$ (b) 782 , and (c) $815 \mathrm{~nm}$ from the same surface area as the images shown in Fig. 4. Depth of the topographical image is $105 \mathrm{~nm}$. Contrast of the optical images is (b) $95 \%$ and (c) $90 \%$.

corners. Exploration of this important functionality of SPPBG structures requires a special investigation and is a subject of our further research.

In summary, using near-field microscopy and SPP excitation at the wavelength of $782 \mathrm{~nm}$, we have directly observed strong reflection of the incident SPP by 400-nmperiod triangular lattice structures simultaneously with the SPP attenuation (below 10\% of the average level) inside these structures for both $\Gamma K$ and $\Gamma M$ orientations. Strong deterioration of both the SPP reflection and inhibition of its propagation for larger wavelengths constitute, in our opinion, an unambiguous evidence of the SPPBG effect in the structures under consideration. We have also directly demonstrated SPP guiding (at $782 \mathrm{~nm}$ ) along line defects in the SPPBG structures. Unhindered propagation of SPP fields in the 3.2- $\mu \mathrm{m}$-wide and $18-\mu \mathrm{m}$-long channel in the SPPBG structure has been observed. Further investigations and optimization of the structural parameters will allow substantial improvement of the effects observed and exploration of the SPPBG-based components similar to those suggested for conventional 2D PBG structures [1-10]. Finally, usage of specialized diffraction gratings for coupling of radiation (eventually from optical fibers) into the SPP would open a direct way for realization of integrated SPPBG circuits for general photonics.

The authors thank V.S. Volkov for help in the experimental work. Electron beam lithography has been performed in III-V Nanolab, a joint laboratory between Research Center COM and the Niels Bohr Institute, Copenhagen University. One of us (S. I. B.) gratefully acknowledges the financial support of the Faculty of Engineering and Science (Aalborg University).

*Author to whom correspondence should be addressed: Institute of Physics, Aalborg University, Pontoppidanstræde 103, DK-9220 Aalborg Øst, Denmark.

Electronic address: sergey@physics.auc.dk

[1] J. D. Joannopoulos, R. D. Meade, and J. N. Winn, Photonic Crystals (Princeton Press, Princeton, New Jersey, 1995).

[2] E. Yablonovitch, Phys. Rev. Lett. 58, 2059 (1987).

[3] S. John, Phys. Rev. Lett. 58, 2486 (1987).

[4] A. Mekis et al., Phys. Rev. Lett. 77, 3787 (1996); J. D. Joannopoulos, P. R. Villeneuve, and S. Fan, Nature (London) 386, 143 (1997).

[5] Lin et al., Science 282, 274 (1998).

[6] T. F. Krauss and R. M. De La Rue, Prog. Quantum Electron. 23, 51 (1999); H. Benisty et al., Appl. Surf. Sci. 164, 205 (2000).

[7] O. Painter et al., Science 284, 1819 (1999).

[8] C. J. M. Smith et al., Electron. Lett. 35, 228 (1999).

[9] H. Kosaka et al., Appl. Phys. Lett. 74, 1370 (1999).

[10] T. D. Happ et al., Electron. Lett. 36, 324 (2000).

[11] H. Raether, Surface Plasmons (Springer-Verlag, Berlin, 1988).

[12] S. C. Kitson, W. L. Barnes, and J. R. Sambles, Phys. Rev. Lett. 77, 2670 (1996).

[13] S. I. Bozhevolnyi and F. A. Pudonin, Phys. Rev. Lett. 78, 2823 (1997); S. I. Bozhevolnyi and V. Coello, Phys. Rev. B 58, 10899 (1998).

[14] DME-DualScope ${ }^{\mathrm{TM}}$, Herlev, Denmark.

[15] B. Hecht et al., J. Appl. Phys. 81, 2492 (1997); S.I. Bozhevolnyi, J. Opt. Soc. Am. B 14, 2254 (1997).

[16] A. V. Shchegrov, I. V. Novikov, and A. A. Maradudin, Phys. Rev. Lett. 78, 4269 (1997). 